\title{
Verifiable Adaptive Control with Analytical Stability Margins by Optimal Control Modification
}

\author{
Nhan T. Nguyen* \\ NASA Ames Research Center, Moffett Field, CA 94035
}

\begin{abstract}
This paper presents a verifiable model-reference adaptive control method based on an optimal control formulation for linear uncertain systems. A predictor model is formulated to enable a parameter estimation of the system parametric uncertainty. The adaptation is based on both the tracking error and predictor error. Using a singular perturbation argument, it can be shown that the closed-loop system tends to a linear time invariant model asymptotically under an assumption of fast adaptation. A stability margin analysis is given to estimate a lower bound of the time delay margin using a matrix measure method. Using this analytical method, the free design parameter $v$ of the optimal control modification adaptive law can be determined to meet a specification of stability margin for verification purposes.
\end{abstract}

\section{Introduction}

Adaptive control is a promising technology that can improve performance and stability of an uncertain system. The ability to accommodate system uncertainties and to improve fault tolerance of a control system is a major selling point of adaptive control since traditional gain-scheduling or fixed-gain controllers are viewed as being less capable of handling systems with uncertainty. In spite of the advances made in the field of adaptive control, there are several challenges related to the implementation of adaptive control technology in safety-critical systems. The absence of the verification and validation methods of adaptive control systems remain a major hurdle to the implementation of adaptive control in safety-critical systems. ${ }^{1,2}$ This hurdle can be traced to the lack of performance and stability metrics for adaptive control which poses a major challenge that prevents adaptive control from being implemented in safety critical systems. The development of verifiable metrics for adaptive control will be important in order to mature adaptive control technology for use in operational safety-critical systems. Of these, stability metrics of adaptive control are an important consideration for assessing system robustness to unmodeled dynamics and exogenous disturbances. In one aspect of verification and validation, a control system is usually certified by demonstrating that it meets an acceptable set of requirements or specifications for stability margins, among other things. Herein lies a major challenge for verification and validation as there is no existing standard tool for stability margin analysis of nonlinear adaptive control. The lack of stability metrics for adaptive control is viewed as a technology barrier to developing certifiable adaptive control for safety-critical systems. ${ }^{1,2}$

Classical LTI control systems are certified by demonstrating that they meet specifications for stability margins among other things. Typically, certification requirements for flight control systems, such as MIL-F-9490D, are often addressed in terms of phase and gain margins. These margins are used for LTI control laws to provide robustness or safety margins in a control system design as a safeguard against unmodeded effects and unstructured uncertainty. While the gain margin concept has been extended to adaptive control, ${ }^{3}$ the phase margin concept does not easily lend itself to adaptive systems due to the inherent nonlinearity in adaptive control. Strictly speaking, phase margin for adaptive control in a global context as in the LTI framework is not possible, but it may be possible to approximate a phase margin locally using linearization or other equivalent methods. ${ }^{4}$ Time delay margin has been viewed as a more readily accepted metric for relative stability of nonlinear control. While time delay margin is a suitable stability metric for adaptive control, a current challenge is that there is no well-established analytical tool for computing the time delay margin.

Verifiable adaptive control must be able to provide measures of stability margins and performance metrics by analytical means. The presence of analytical methods for analyzing stability and performance of an adaptive control law will enable a designer to conduct the design of an adaptive controller using an analytical approach as opposed to

${ }^{*}$ Research Scientist, Intelligent Systems Division, Mail Stop 269-1, AIAA Associate Fellow 
an ad-hoc trial-and-error process that is typically done. Currently, there is no adaptive control method that can provide any analytical stability margin for nonlinear uncertain systems. On the other hand, for linear uncertain systems, the $\mathscr{L}_{1}$ adaptive control method can provide both stability and performance measures under the assumption of fast adaptation. ${ }^{5,6}$ The stability margin of the $\mathscr{L}_{1}$ adaptive control is then governed by the choice of a low-pass filter implementation.

This paper presents another possibility for verifiable adaptive control based on the use of the optimal control modification adaptive law that was recently developed. ${ }^{7}$ This method provides a modification to model-reference adaptive control to improve robustness to unmodeled dynamics and other unknown disturbances. The adaptive law was derived from the optimal control theory to minimize the $\mathscr{L}_{2}$-norm of the tracking error. It should be mentioned that there are other recently developed modifications to adaptive control such as the adaptive loop recovery method, ${ }^{8}$ Kalman filter modification, ${ }^{9}$ and $Q$-modification. ${ }^{10}$

In the context of verifiable adaptive control, the optimal control modification exhibits an interesting property. Using a singular perturbation argument for fast adaptation, it can be shown that the asymptotic solution of the adaptive law for a linear uncertain system actually tends to a LTI model. This asymptotic linearity affords a certain advantage that can allow analytical methods to be developed for analyzing stability margins. This behavior will be explored in this paper. The design approach for linear uncertain systems developed herein is based on a combined adaptation to minimize both the tracking error and predictor error. A number of adaptive control methods that utilize predictor models include the $\mathscr{L}_{1}$ adaptive control, composite model-reference adaptive control, ${ }^{11}$ and $\mathscr{H}_{\infty}$ adaptive control. ${ }^{12}$ The predictor model is utilized to enabled the control input uncertainty to be estimated. Under fast adaptation, the optimal control modification provides an analytical expression to compute the minimum time delay margin of the system that is lower-bounded by a non-zero value. This behavior is very similar to the $\mathscr{L}_{1}$ adaptive control even though the approach is entirely and uniquely different.

\section{Optimal Control Modification Adaptive Law}

Consider a linear plant with control input uncertainty and linear matched uncertainty

$$
\dot{x}=A x+B \Lambda\left(u+\Omega^{* \top} x\right)
$$

where $x(t):[0, \infty) \rightarrow \mathbb{R}^{n}$ is a state vector, $u(t):[0, \infty) \rightarrow \mathbb{R}^{p}$ is a control vector, $A \in \mathbb{R}^{n \times n}$ and $B \in \mathbb{R}^{n \times p}, p \geq n$, are constant and known matrices such that the pair $(A, B)$ is controllable, $\Lambda=\Lambda^{\top}>0 \in \mathbb{R}^{p \times p}$ is a constant and unknown diagonal matrix with positive diagonal elements, and $\Omega^{*} \in \mathbb{R}^{n \times p}$ is a constant and unknown matrix that represents a matched parametric uncertainty..

A nominal fixed gain controller has been designed to stabilize the nominal plant with $\Lambda=I$ and $\Omega^{*}=0$, and to enable it to track a command $r(t)$

$$
u_{\text {nom }}=-K_{x} x+K_{r} r
$$

where $r(t):[0, \infty) \rightarrow \mathbb{R}^{r} \in \mathscr{L}_{\infty}$ is a piecewise-continuous and bounded command vector, $A-B K_{x} \in \mathbb{R}^{n \times n}$ is Hurwitz, and $B K_{r} \in \mathbb{R}^{n \times r}, r \leq n$.

The closed-loop nominal plant without uncertainty is

$$
\dot{x}=A_{m} x+B_{m} r
$$

This closed-loop nominal plant is then used to specify a reference model

$$
\dot{x}_{m}=A_{m} x_{m}+B_{m} r
$$

where $x_{m}(t):[0, \infty) \rightarrow \mathbb{R}^{n}$ is a reference state vector, and $A_{m}=A-B K_{x}$ and $B_{m}=B K_{r}$.

Since $r(t)$ is bounded, then $x_{m}(t)$ can be shown to be uniformly bounded such that

$$
\left\|x_{m}(0)\right\|<\varepsilon \Rightarrow\left\|x_{m}(t)\right\| \leq \delta(\varepsilon), \forall t \geq 0
$$

The objective is to design a full-state feedback adaptive augmentation controller to enable $x(t)$ to follow $x_{m}(t)$ in the presence of uncertainty due to $\Lambda$ and $\Omega^{*}$ with the following controller

$$
u=u_{\text {nom }}-\Delta K_{x} x+\Delta K_{r} r-\Omega^{\top} x
$$

where $\Delta K_{x}(t):[0, \infty) \rightarrow \mathbb{R}^{p \times n}, \Delta K_{r}(t):[0, \infty) \rightarrow \mathbb{R}^{p \times r}$, and $\Omega(t):[0, \infty) \rightarrow \mathbb{R}^{n \times p}$. 
For convenience. $u(t)$ can be expressed as

$$
u=u_{n o m}-\Theta^{\top} \Phi
$$

where $\Theta^{\top}=\left[\begin{array}{cc}\Delta K_{x}+\Omega^{\top} & -\Delta K_{r}\end{array}\right]$ and $\Phi=\left[\begin{array}{ll}x^{\top} & r^{\top}\end{array}\right]^{\top}$.

Assumption 1: There exist constant and unknown $\Delta K_{x}^{*}$ and $\Delta K_{r}^{*}$ such that the following conditions are satisfied

$$
\begin{aligned}
& \Lambda\left(K_{x}+\Delta K_{x}^{*}\right)=K_{x} \\
& \Lambda\left(K_{r}+\Delta K_{r}^{*}\right)=K_{r}
\end{aligned}
$$

Let $\tilde{\Lambda}=\hat{\Lambda}-\Lambda, \Delta \tilde{K}_{r}=\Delta K_{r}-\Delta K_{r}^{*}, \Delta \tilde{K}_{x}=\Delta K_{x}-\Delta K_{x}^{*}$, and $\tilde{\Omega}=\Omega-\Omega^{*}$. Then the closed-loop plant becomes

$$
\dot{x}=A_{m} x+B_{m} r+B \hat{\Lambda}\left(-\Delta \tilde{K}_{x} x+\Delta \tilde{K}_{r} r-\tilde{\Omega}^{\top} x\right)-B \tilde{\Lambda}\left(-\Delta \tilde{K}_{x} x+\Delta \tilde{K}_{r} r-\tilde{\Omega}^{\top} x\right)
$$

or

$$
\dot{x}=A_{m} x+B_{m} r-B \hat{\Lambda} \tilde{\Theta}^{\top} \Phi+B \tilde{\Lambda} \tilde{\Theta}^{\top} \Phi
$$

Defining the tracking error as $e(t)=x_{m}(t)-x(t)$, then the tracking error equation becomes

$$
\dot{e}=A_{m} e+B \hat{\Lambda} \tilde{\Theta}^{\top} \Phi+B \varepsilon
$$

where $\varepsilon(x):: \mathbb{R}^{n} \rightarrow \mathbb{R}^{n}$ is the estimation error

$$
\varepsilon=-\tilde{\Lambda} \tilde{\Theta}^{\top} \Phi
$$

The standard MRAC adaptive law for adjusting $\Theta$ is given as

$$
\dot{\Theta}=-\Gamma_{\Theta} \Phi e^{\top} P B \operatorname{sgn} \Lambda
$$

This MRAC law, while providing asymptotic tracking, does not provide robustness to potential unmodeled dynamics. Optimal control modification adaptive law has been developed to address robustness and can be used to provide the adaptation as follows:

$$
\dot{\Theta}=-\Gamma_{\Theta} \Phi\left(e^{\top} P-v \Phi^{\top} \Theta \hat{\Lambda}^{\top} B^{\top} P A_{m}^{-1}\right) B \hat{\Lambda}
$$

It is noted that the adaptive law depends the estimate of $\Lambda$ which needs to be computed. Toward this end, a predictor model of the plant is defined as

$$
\begin{gathered}
\dot{\hat{x}}=A_{m} \hat{x}+\left(A-A_{m}\right) \hat{x}+B \hat{\Lambda}\left(u+\Omega^{\top} x\right) \\
\dot{x}=A x+B \Lambda\left(u+\Omega^{\top} x-\tilde{\Omega}^{\top} x\right)
\end{gathered}
$$

Defining the predictor error as $e_{p}(t)=\hat{x}(t)-x(t)$, then where

$$
\varepsilon_{p}=-\tilde{\Lambda} \tilde{\Omega}^{\top} x
$$

Proposition 1: The following adaptive laws

$$
\begin{gathered}
\dot{\Omega}=-\Gamma_{\Omega} x\left(e_{p}^{\top} P-v x^{\top} \Omega \hat{\Lambda}^{\top} B^{\top} P A_{m}^{-1}\right) B \hat{\Lambda} \\
\dot{\hat{\Lambda}}^{\top}=-\Gamma_{\Lambda}\left(u+\Omega^{\top} x\right)\left[e_{p}^{\top} P-v\left(u^{\top}+x^{\top} \Omega\right) \hat{\Lambda}^{\top} B^{\top} P A_{m}^{-1}\right] B
\end{gathered}
$$

is an approximate solution of an optimal control problem that minimizes the following an infinite-time horizon cost function

$$
J=\lim _{t_{f} \rightarrow \rightarrow \infty} \frac{1}{2} \int_{0}^{t_{f}}\left(e_{p}-\Delta\right)^{\top} Q\left(e_{p}-\Delta\right) d t
$$

where $\Delta(t):[0, \infty) \rightarrow \mathbb{R}^{n}$ is a lower bound of the tracking error, $\Gamma_{\Omega}=\Gamma_{\Omega}^{\top}>0 \in \mathbb{R}^{n \times n}$ and $\Gamma_{\Lambda}=\Gamma_{\Lambda}^{\top}>0 \in \mathbb{R}^{p \times p}$ are adaptive gain matrices, $v>0 \in \mathbb{R}$ is a free design parameter, and $P=P^{\top}>0 \in \mathbb{R}^{n \times n}$ that solves

$$
P A_{m}+A_{m}^{\top} P=-Q
$$


where $Q=Q^{\top}>0 \in \mathbb{R}^{n \times n}$.

Proof: The cost function $J$ is convex and represents the distance measured from a point on the trajectory of $e_{p}(t)$ to the normal surface of a hypersphere $B_{\Delta}=\left\{e_{p}(t) \in \mathbb{R}^{n}:\left\|e_{p}(t)\right\| \leq\|\Delta\|\right\} \subset \mathscr{D} \subset \mathbb{R}^{n}$. The cost function is designed to provide robustness by not seeking an asymptotic predictor error that tends to zero but rather one that tends to some lower bound away from the origin. By not requiring $e_{p}(t) \rightarrow 0$ as $t \rightarrow \infty$, the adaptation can be made to be robust. Therefore, the tracking performance can be traded with robustness by a suitable selection of the tuning parameter $v$.

An optimal control problem can be formulated by the Pontryagin's Minimum Principle. Define a Hamiltonian function

$$
H\left(e_{p}, p, \tilde{\Lambda}, \tilde{\Omega}\right)=\frac{1}{2}\left(e_{p}-\Delta\right)^{\top} Q\left(e_{p}-\Delta\right)+p^{\top}\left[A_{m} e_{p}+B \tilde{\Lambda}\left(u+\Omega^{\top} x\right)+B \hat{\Lambda} \tilde{\Omega}^{\top} x+B \varepsilon_{p}\right]
$$

where $p(t):[0, \infty) \rightarrow \mathbb{R}^{n}$ is an adjoint variable, then the adjoint equation can be established by the following necessary condition

$$
\dot{p}=-\nabla H_{e_{p}}^{\top}=-Q\left(e_{p}-\Delta\right)-A_{m}^{\top} p
$$

with the transversality condition $p\left(t_{f} \rightarrow \infty\right)=0$ since $e(0)$ is known.

Then the adaptive laws can be formulated by a gradient method as ${ }^{13}$

$$
\begin{gathered}
\dot{\tilde{\Omega}}=-\Gamma_{\Omega} \nabla H_{\tilde{\Omega}^{\top}}=-\Gamma_{\Omega} x p^{\top} B \hat{\Lambda} \\
\dot{\tilde{\Lambda}}^{\top}=-\Gamma_{\Lambda} \nabla H_{\tilde{\Lambda}}=-\Gamma_{\Lambda}\left(u+\Omega^{\top} x\right) p^{\top} B
\end{gathered}
$$

An "approximate" solution of $p(t)$ is obtained using a "sweeping" method ${ }^{14}$ for the adaptive law for $\Omega$ by letting $p=P e_{p}+S B \hat{\Lambda} \Omega^{\top} x$. Then, the adjoint equation becomes

$$
\begin{aligned}
\dot{P} e_{p}+P\left[A_{m} e_{p}+B \tilde{\Lambda}\left(u+\Omega^{\top} x\right)+B \hat{\Lambda}\left(\Omega^{\top} x-\Omega^{* \top} x\right)+B \varepsilon\right]+\dot{S} B \hat{\Lambda} \Omega^{\top} x+S B \frac{d\left(\Omega^{\top} x\right)}{d t} & \\
& =-Q\left(e_{p}-\Delta\right)-A_{m}^{\top}\left[P e_{p}+S B \hat{\Lambda} \Omega^{\top} x\right]
\end{aligned}
$$

which yields steady-state constant solutions of $P$ and $S$ as

$$
\begin{gathered}
P A_{m}+A_{m}^{\top} P+Q=0 \\
S=-A_{m}^{-\top} P
\end{gathered}
$$

Without any loss of generality, a free design parameter $v>0 \in \mathbb{R}$ is introduced as a gain to allow for adjustments of the modification term in the adaptive law, where $v=1$ corresponds to an optimal solution. Thus

$$
S=-v A_{m}^{-\top} P
$$

Then the adjoint $p$ is obtained as

$$
p=P e_{p}-v A_{m}^{-\top} P B \hat{\Lambda} \Omega^{\top} x
$$

Substituting $p$ into the gradient adaptive laws yields the adaptive law (19). The derivation of the adaptive law (80) can be done in a similar manner.

Theorem 1: The adaptive laws (19) and(80) result in stable and uniformly ultimately bounded predictor error $e_{p}(t)$ for all $\left(e_{p}(0), \tilde{\Omega}(0), \tilde{\Lambda}(0)\right) \in B_{\alpha}$ with an ultimate bound

$$
\rho=\sqrt{\frac{\lambda_{\max }(P) r^{2}+\lambda_{\max }\left(\Gamma_{\Omega}^{-1}\right) \kappa^{2}+\lambda_{\max }\left(\Gamma_{\Lambda}^{-1}\right) v^{2}}{\lambda_{\min }(P)}}
$$

where

$$
\begin{gathered}
r=\frac{2\|P B\| \varepsilon_{0}}{\lambda_{\min }(Q)} \\
\kappa=\frac{2\left\|P A_{m}^{-1}\right\| \Omega_{0}}{\lambda_{\min }\left(A_{m}^{-\top} Q A_{m}^{-1}\right)}
\end{gathered}
$$




$$
v=\frac{2\left\|P A_{m}^{-1}\right\| \Lambda_{0}}{\lambda_{\min }\left(A_{m}^{-\top} Q A_{m}^{-1}\right)}
$$

with $\varepsilon_{0}=\sup _{x \in \mathscr{D}}\left\|\varepsilon_{p}\right\|, \Omega_{0}=\max \left\|\Omega^{*}\right\|$, and $\Lambda_{0}=\max \|\Lambda\|$.

Proof: Choose a Lyapunov candidate function

$$
V=e_{p}^{\top} P e_{p}+\operatorname{trace}\left(\tilde{\Omega}^{\top} \Gamma_{\Omega}^{-1} \tilde{\Omega}\right)+\operatorname{trace}\left(\tilde{\Lambda} \Gamma_{\Lambda}^{-1} \tilde{\Lambda}^{\top}\right)
$$

Evaluating $\dot{V}$ yields

$$
\begin{aligned}
\dot{V}=e_{p}^{\top}\left(A_{m} P+P A_{m}\right) e_{p}+2 e_{p}^{\top} P\left[B \tilde{\Lambda}\left(u+\Omega^{\top} x\right)+B \hat{\Lambda} \tilde{\Omega}^{\top} x+B \varepsilon_{p}\right]-2 \operatorname{trace}\left[\tilde{\Omega}^{\top} x e_{p}^{\top} P B \hat{\Lambda}-v \tilde{\Omega}^{\top} x x^{\top} \Omega \hat{\Lambda}^{\top} B^{\top} P A_{m}^{-1} B \hat{\Lambda}\right] \\
-2 \operatorname{trace}\left[\tilde{\Lambda}\left(u+\Omega^{\top} x\right) e_{p}^{\top} P B-v \tilde{\Lambda}\left(u+\Omega^{\top} x\right)\left(u^{\top}+x^{\top} \Omega\right) \hat{\Lambda}^{\top} B^{\top} P A_{m}^{-1} B\right]
\end{aligned}
$$

Using the trace identity trace $\left(A^{\top} B\right)=B A^{\top}, \dot{V}$ can be written as

$$
\begin{aligned}
\dot{V}=-e_{p}^{\top} Q e_{p}+2 e_{p}^{\top} P\left[B \tilde{\Lambda}\left(u+\Omega^{\top} x\right)\right. & \left.+B \hat{\Lambda} \tilde{\Omega}^{\top} x+B \varepsilon_{p}\right]-2 e_{p}^{\top} P B \hat{\Lambda} \tilde{\Omega}^{\top} x+2 v x^{\top} \Omega \hat{\Lambda}^{\top} B^{\top} P A_{m}^{-1} B \hat{\Lambda} \tilde{\Omega}^{\top} x \\
& -2 e_{p}^{\top} P B \tilde{\Lambda}\left(u+\Omega^{\top} x\right)+2 v\left(u^{\top}+x^{\top} \Omega\right) \hat{\Lambda}^{\top} B^{\top} P A_{m}^{-1} B \tilde{\Lambda} \tilde{\Lambda}\left(u+\Omega^{\top} x\right)
\end{aligned}
$$

The sign-definiteness of the term $P A_{m}^{-1}$ is now considered. Recall that a general real matrix $G$ is positive (negative) definite if and only if its symmetric part $M=\frac{1}{2}\left(G+G^{\top}\right)$ is also positive (negative) definite. Then, by pre- and postmultiplication of Eq. (22) by $A_{m}^{-\top}$ and $A_{m}^{-1}$, respectively, $P A_{m}^{-1}$ can be decomposed into a symmetric part $M$ and anti-symmetric part $N$ as

$$
P A_{m}^{-1}=M+N
$$

where

$$
\begin{gathered}
M=\frac{1}{2}\left(A_{m}^{-\top} P+P A_{m}^{-1}\right)=-\frac{1}{2} A_{m}^{-\top} Q A_{m}^{-1} \\
N=\frac{1}{2}\left(P A_{m}^{-1}-A_{m}^{-\top} P\right) .
\end{gathered}
$$

Since the symmetric part $M<0$, then $P A_{m}^{-1}<0$. Thus, $\dot{V}$ becomes

$$
\begin{aligned}
\dot{V}=-e_{p}^{\top} Q e_{p}+2 e_{p}^{\top} P B \varepsilon_{p}+2 v x^{\top}\left(\Omega^{*}+\tilde{\Omega}\right) \hat{\Lambda}^{\top} B^{\top}(M+N) B \hat{\Lambda} \tilde{\Omega}^{\top} x \\
+2 v\left(u^{\top}+x^{\top} \Omega\right)(\Lambda+\tilde{\Lambda})^{\top} B^{\top}(M+N) B \tilde{\Lambda}\left(u+\Omega^{\top} x\right)
\end{aligned}
$$

Using the property $y^{\top} N y=0$ for an anti-symmetric matrix $N, \dot{V}$ is reduced to

$$
\begin{aligned}
\dot{V}=-e_{p}^{\top} Q e_{p} & +2 e_{p}^{\top} P B \varepsilon_{p}+2 v x^{\top} \Omega^{*} \hat{\Lambda}^{\top} B^{\top} P A_{m}^{-1} B \hat{\Lambda} \tilde{\Omega}^{\top} x-v x^{\top} \tilde{\Omega} \hat{\Lambda}^{\top} B^{\top} A_{m}^{-\top} Q A_{m}^{-1} B \hat{\Lambda}^{\top} \tilde{\Omega}^{\top} x \\
& +2 v\left(u^{\top}+x^{\top} \Omega\right) \Lambda^{\top} B^{\top} P A_{m}^{-1} B \tilde{\Lambda}\left(u+\Omega^{\top} x\right)-v\left(u^{\top}+x^{\top} \Omega\right) \tilde{\Lambda}^{\top} B^{\top} A_{m}^{-\top} Q A_{m}^{-1} B \tilde{\Lambda}\left(u+\Omega^{\top} x\right)
\end{aligned}
$$

which is bounded by

$$
\begin{aligned}
\dot{V} \leq-\left\|e_{p}\right\|\left[\lambda_{\min }(Q)\left\|e_{p}\right\|-2\|P B\| \varepsilon_{0}\right] & -v\|x\|^{2}\|B \hat{\Lambda}\|^{2}\|\tilde{\Omega}\|\left[\lambda_{\min }\left(A_{m}^{-\top} Q A_{m}^{-1}\right)\|\tilde{\Omega}\|-2\left\|P A_{m}^{-1}\right\| \Omega_{0}\right] \\
& -v\left\|u+\Omega^{\top} x\right\|^{2}\|B\|^{2}\|\tilde{\Lambda}\|\left[\lambda_{\min }\left(A_{m}^{-\top} Q A_{m}^{-1}\right)\|\tilde{\Lambda}\|-2\left\|P A_{m}^{-1}\right\| \Lambda_{0}\right]
\end{aligned}
$$

Let

$$
B_{r}=\left\{\left(e_{p}, \tilde{\Omega}, \tilde{\Lambda}\right) \in \mathbb{R}^{n} \times \mathbb{R}^{n \times p} \times \mathbb{R}^{p \times p}:\left\|e_{p}\right\| \leq r \text { or }\|\tilde{\Omega}\| \leq \kappa \text { or }\|\tilde{\Lambda}\| \leq v\right\}
$$

where

$$
\left\|e_{p}\right\| \leq r=\frac{2\|P B\| \varepsilon_{0}}{\lambda_{\min }(Q)}
$$




$$
\begin{gathered}
\|\tilde{\Omega}\| \leq \kappa=\frac{2\left\|P A_{m}^{-1}\right\| \Omega_{0}}{\lambda_{\min }\left(A_{m}^{-\top} Q A_{m}^{-1}\right)} \\
\|\tilde{\Lambda}\| \leq v=\frac{2\left\|P A_{m}^{-1}\right\| \Lambda_{0}}{\lambda_{\min }\left(A_{m}^{-\top} Q A_{m}^{-1}\right)}
\end{gathered}
$$

It follows that $\dot{V} \leq 0$ for all $\left(e_{p}, \tilde{\Omega}, \tilde{\Lambda}\right) \in B_{R}-B_{r}$, where $B_{R}=\left\{e_{p} \in \mathbb{R}^{n}:\left\|e_{p}\right\| \leq R\right\} \subset \mathscr{D}$. Let $B_{\beta}$ be the smallest subset that encloses $B_{r}$, then there exists $\beta>0$ where

$$
\beta=\lambda_{\max }(P) r^{2}+\lambda_{\max }\left(\Gamma_{\Omega}^{-1}\right) \kappa^{2}+\lambda_{\max }\left(\Gamma_{\Lambda}^{-1}\right) v^{2}
$$

such that

$$
B_{r} \subset B_{\beta}=\left\{\left(e_{p}, \tilde{\Omega}, \tilde{\Lambda}\right) \in \mathbb{R}^{n} \times \mathbb{R}^{n \times p} \times \mathbb{R}^{p \times p}: V \leq \beta\right\}
$$

Let $B_{\alpha}$ be the largest subset enclosed by $B_{R}$, then since $\left\|e_{p}\right\| \leq R$ in $B_{R}$, there exists $\alpha>0$ where

$$
\lambda_{\text {min }}(P)\left\|e_{p}\right\|^{2} \leq \lambda_{\min }(P)\left\|e_{p}\right\|^{2}+\lambda_{\text {min }}\left(\Gamma_{\Omega}^{-1}\right)\|\tilde{\Omega}\|^{2}+\lambda_{\min }\left(\Gamma_{\Lambda}^{-1}\right)\|\tilde{\Lambda}\|^{2} \leq V \leq \lambda_{\min }(P) R^{2}=\alpha
$$

such that

$$
B_{\alpha}=\left\{\left(e_{p}, \tilde{\Omega}, \tilde{\Lambda}\right) \in \mathbb{R}^{n} \times \mathbb{R}^{n \times p} \times \mathbb{R}^{p \times p}: V \leq \alpha\right\} \subset B_{R}
$$

Then for a solution to be uniformly bounded, the set containment is as follows:

$$
B_{r} \subset B_{\beta} \subset B_{\alpha} \subset B_{R}
$$

This implies

$$
\beta<\alpha \Leftrightarrow \lambda_{\max }(P) r^{2}+\lambda_{\max }\left(\Gamma_{\Omega}^{-1}\right) \kappa^{2}+\lambda_{\max }\left(\Gamma_{\Lambda}^{-1}\right) v^{2}<\lambda_{\min }(P) R^{2}
$$

Therefore

$$
R>\sqrt{\frac{\lambda_{\max }(P) r^{2}+\lambda_{\max }\left(\Gamma_{\Omega}^{-1}\right) \kappa^{2}+\lambda_{\max }\left(\Gamma_{\Lambda}^{-1}\right) v^{2}}{\lambda_{\min }(P)}}=\rho
$$

where $\rho$ is the smallest value of $R$.

Then $\rho$ is the ultimate bound of $e_{p}(t)$ such that

$$
r \leq\left\|e_{p}(t)\right\| \leq \rho \leq R
$$

Since $\dot{V} \leq 0$ for all $\left(e_{p}, \tilde{\Omega}, \tilde{\Lambda}\right) \in B_{R}-B_{r}$, therefore $V$ is a decreasing function of time outside of $B_{r}$. Thus, if $\left(e_{p}(0), \tilde{\Omega}(0), \tilde{\Lambda}(0)\right) \in B_{\alpha}$, the solution will eventually enters $B_{\beta}$ after a finite time $t=T$ (independent of $\left(e_{p}(0), \tilde{\Omega}(0), \tilde{\Lambda}(0)\right)$ and $\alpha$ ) and remain inside for all $t>T .{ }^{15}$ Therefore, $e_{p}(t)$ is uniformly ultimately bounded with an ultimate bound $\rho$.

\section{Transient and steady state performance}

It can easily be shown using the Lyapunov analysis above that the tracking error is bounded by

$$
\|e(t)\|_{\mathscr{L}_{\infty}} \leq \sqrt{\frac{\lambda_{\max }(P) w^{2}+\lambda_{\max }\left(\Gamma_{\Theta}^{-1}\right) \sigma^{2}}{\lambda_{\min }(P)}}
$$

where

$$
\begin{gathered}
w=\frac{2\|P B\| \sup _{x \in \mathscr{D}}\|\varepsilon\|}{\lambda_{\min }(Q)} \\
\sigma=\frac{2\left\|P A_{m}^{-1}\right\| \max \left\|\Theta^{*}\right\|}{\lambda_{\min }\left(A_{m}^{-\top} Q A_{m}^{-1}\right)}
\end{gathered}
$$

Thus, $\|e(t)\|_{\mathscr{L}_{\infty}} \propto \sqrt{\lambda_{\max }\left(\Gamma_{\Theta}^{-1}\right)}$. Increasing $\Gamma_{\Theta}$ gives better transient and steady state tracking performance. However, that will come at the expense of robustness. Optimal control modification adaptive laws provide stability margins to improve robustness even when $\Gamma_{\Theta}$ is large. 
Similarly, the predictor error is bounded by

$$
\left\|e_{p}(t)\right\|_{\mathscr{L}_{\infty}}=\sqrt{\frac{\lambda_{\max }(P) r^{2}+\lambda_{\max }\left(\Gamma_{\Omega}^{-1}\right) \kappa^{2}+\lambda_{\max }\left(\Gamma_{\Lambda}^{-1}\right) v^{2}}{\lambda_{\min }(P)}}
$$

Increasing the adaptive gains $\Gamma_{\Omega}$ and $\Gamma_{\Lambda}$ results in better convergence of the parameter estimation of $\Omega^{*}$ and $\Lambda$. With optimal control modification, the adaptive gains can be set as large as numerically feasible to obtain better parameter convergence without adversely affecting stability robustness of the parameter estimation algorithms.

Numerically speaking, if the Euler integration method is implemented with a time step $\Delta t$, there exists a maximum value of $\Gamma_{\Theta}$ to maintain numerical stability. This is a well-known result in numerical analysis. For the tracking error adaptation, an approximate numerical limit of $\Gamma_{\Theta}$ based on a given sampling frequency $f_{s}$ is given by

$$
\lambda_{\max }\left(\Gamma_{\Theta}\right) \leq \frac{2 f_{s}}{v\left\|\Phi \Phi^{\top}\right\|_{\mathscr{L}_{\infty}}\left\|\hat{\Lambda}^{\top} B^{\top} P A_{m}^{-1} B \hat{\Lambda}\right\|_{\mathscr{L}_{\infty}}}
$$

The norm $\left\|\Phi \Phi^{\top}\right\|_{\mathscr{L}_{\infty}}$ can be estimated based on $x_{m}$ instead of $x$, but this would tend to yield a non-conservative estimate. Similarly, the norm $\left\|\hat{\Lambda}^{\top} B^{\top} P A_{m}^{-1} B \hat{\Lambda}\right\|_{\mathscr{L}_{\infty}}$ can be approximated by $\left\|B^{\top} P A_{m}^{-1} B\right\|_{\mathscr{L}_{\infty}}$ which is a conservative estimate.

\section{Stability Margins}

The tracking error equation can also be written as

$$
\dot{e}=A_{m} e+B \hat{\Lambda} \Theta^{\top} \Phi-B \Lambda \Theta^{* \top} \Phi-B \tilde{\Lambda} \Theta^{\top} \Phi
$$

Now consider a time-scale separation problem when $\left\|\Gamma_{\Theta}\right\|=\frac{\gamma_{\Theta}}{\varepsilon} \gg\left\|A_{m}\right\|$ where $\varepsilon$ is a small parameter. Then $\Theta(t)$ is said to be a fast state and $e(t)$ is a slow state. To decouple the fast and slow states, a time-scale separation can be performed by applying the singular perturbation method using a time transformation

$$
\tau=\varepsilon t
$$

where $\tau$ is a slow time variable.

Then, the system is transformed into a singularly perturbed system as

$$
\begin{gathered}
\frac{d e}{d \tau}=\frac{1}{\varepsilon}\left[A_{m} e+B \hat{\Lambda} \Theta^{\top} \Phi-B \Lambda \Theta^{* \top} \Phi-B \tilde{\Lambda} \Theta^{\top} \Phi\right] \\
\varepsilon \frac{d \Theta}{d \tau} \approx-\frac{\gamma_{\Theta}}{\varepsilon} \Phi\left(e^{\top} P-v \Phi^{\top} \Theta \hat{\Lambda}^{\top} B^{\top} P A_{m}^{-1}\right) B \hat{\Lambda}
\end{gathered}
$$

The Tikhonov's theorem can be used to approximate the solution of the singularly perturbed system with the solution of a "reduced-order" system by letting $\varepsilon \rightarrow 0 .{ }^{16}$ Then, $\Theta(e, \varepsilon)$ is on a fast manifold. Thus, the reduced-order system is given by

$$
\begin{gathered}
\varepsilon^{2} \frac{d \Theta}{d \tau} \approx-\gamma_{\Theta} \Phi\left(e^{\top} P-v \Phi^{\top} \Theta \hat{\Lambda}^{\top} B^{\top} P A_{m}^{-1}\right) B \hat{\Lambda} \Rightarrow\left(B \hat{\Lambda} \Theta^{\top} \Phi\right)^{(0)}=\frac{1}{v} P^{-1} A_{m}^{\top} P e^{(0)} \\
\dot{e}^{(0)}=A_{m} e^{(0)}+\left(B \hat{\Lambda} \Theta^{\top} \Phi\right)^{(0)}-\left(B \Lambda \Theta^{* \top} \Phi\right)^{(0)}-\left(B \tilde{\Lambda} \Theta^{\top} \Phi\right)^{(0)}
\end{gathered}
$$

where $e^{(0)}(t)$ and $\left(\hat{B} \Theta^{\top} \Phi\right)^{(0)}(t)$ are the "outer" solutions of the singularly perturbed system.

The "inner" or "boundary layer" solution for this system is obtained from

$$
\begin{gathered}
\dot{\Theta}^{(i)}=-\Gamma_{\Theta} \Phi^{(i)}\left[e^{(i) \top} P-v\left(\Phi^{\top} \Theta \hat{\Lambda}^{\top} B^{\top}\right)^{(i)} P A_{m}^{-1}\right] B \hat{\Lambda}^{(i)} \\
\varepsilon \frac{d e}{d \tau}=A_{m} e+B \hat{\Lambda} \Theta^{\top} \Phi-B \Lambda \Theta^{* \top} \Phi-B \tilde{\Lambda} \Theta^{\top} \Phi \Rightarrow A_{m} e^{(i)}+\left(B \hat{\Lambda} \Theta^{\top} \Phi\right)^{(i)}-\left(B \Lambda \Theta^{* \top} \Phi\right)^{(i)}-\left(B \tilde{\Lambda} \Theta^{\top} \Phi\right)^{(i)}=0
\end{gathered}
$$


The general solution of the system is then expressed as

$$
e(t)=e^{(0)}(t)+e^{(i)}(t)-e_{M A E}(t)
$$

where $e_{M A E}(t)$ is a correction term by a matched asymptotic expansion method applied to both the inner and outer solutions. ${ }^{17}$ The outer solution is in fact the asymptotic solution of the original system as $t \rightarrow \infty$. For the singularly perturbed system comprising $e^{(0)}(t)$ and $\left(\hat{B} \Theta^{\top} \Phi\right)^{(0)}(t)$, the outer solution of the tracking error is then determined by

$$
\dot{e}^{(0)}=\left(A_{m}+\frac{1}{v} P^{-1} A_{m}^{\top} P\right) e^{(0)}+B \Lambda\left(-\Delta K_{x}^{*} x+\Delta K_{r}^{*} r-\Omega^{* \top} x\right)+\Delta
$$

where $\Delta$ is the residual uncertainty

$$
\Delta=B \tilde{\Lambda}\left(-\Delta K_{x} x+\Delta K_{r} r-\Omega^{\top} x\right)
$$

which interestingly enough is an LTI system.

Since the asymptotic tracking error equation for the outer solution is LTI, many standard LTI analysis tools can be used to estimate the asymptotic behavior of the original system. In particular, the design parameter $v$ can be selected to provide a notion of stability margin for the outer solution to account for the residual uncertainty $\Delta$.

Let $\Delta A=B \Lambda \Omega^{* \top} x$ and $\Delta B=B(\Lambda-I)$, then the plant can also be written as

$$
\dot{x}=(A+\Delta A) x+(B+\Delta B) u
$$

From the model matching conditions, we have

$$
\begin{aligned}
& B \Lambda \Delta K_{x}^{*}=B(I-\Lambda) K_{x}=-\Delta B K_{x} \\
& B \Lambda \Delta K_{r}^{*}=B(I-\Lambda) K_{r}=-\Delta B K_{r}
\end{aligned}
$$

Therefore, the asymptotic tracking error equation becomes

$$
\dot{e}^{(0)}=\left[A+\Delta A-(B+\Delta B) K_{x}+\frac{1}{v} P^{-1} A_{m}^{\top} P\right] e^{(0)}-\left(\Delta A-\Delta B K_{x}\right) x_{m}-\Delta B K_{r} r+\Delta
$$

A similar singular perturbation argument can be applied to the predictor model to obtain an asymptotic predictor model under fast adaptation. The asymptotic predictor model can be shown to be

$$
\dot{e}_{p}^{(0)}=\left(A_{m}+\frac{1}{v} P^{-1} A_{m}^{\top} P-B K_{x}\right) e_{p}^{(0)}+B K x_{m}-B K_{r} r
$$

which is also a LTI model and interestingly enough does not depend on plant uncertainty.

The projection operator can be applied to the adaptive laws with a priori knowledge of the bounds on the elements of $\Theta, \Omega$, and $\hat{\Lambda}$. Using these a priori bounds, the design parameter $v$ can be selected to guarantee stability robustness. Then the adaptive laws are modified by the projection operator as follows:

$$
\begin{gathered}
\dot{\Theta}=-\Gamma_{\Theta} \operatorname{Proj}\left(\Theta, \Phi\left(e^{\top} P-v \Phi^{\top} \Theta \hat{\Lambda}^{\top} B^{\top} P A_{m}^{-1}\right) B \hat{\Lambda}\right) \\
\dot{\Omega}=-\Gamma_{\Omega} \operatorname{Proj}\left(\Omega, x\left(e_{p}^{\top} P-v x^{\top} \Omega \hat{\Lambda}^{\top} B^{\top} P A_{m}^{-1}\right) B \hat{\Lambda}\right) \\
\dot{\hat{\Lambda}}^{\top}=-\Gamma_{\Lambda} \operatorname{Proj}\left(\hat{\Lambda},\left(u+\Omega^{\top} x\right)\left[e_{p}^{\top} P-v\left(u^{\top}+x^{\top} \Omega\right) \hat{\Lambda}^{\top} B^{\top} P A_{m}^{-1}\right] B\right)
\end{gathered}
$$

The free design parameter $v$ can now be estimated to give the asymptotic closed-loop tracking error model a desired measure of stability robustness such as phase or time delay margin to account for the residual uncertainty and any other unmodeled dynamics. One method for estimating a MIMO phase or time delay margin is based on the matrix measure method which is given as follows:

In the presence of an input delay, the closed-loop tracking error becomes

$$
\dot{e}^{(0)}(t)=(A+\Delta A) e^{(0)}(t)-\left[(B+\Delta B) K_{x}-\frac{1}{v} P^{-1} A_{m}^{\top} P\right] e^{(0)}\left(t-t_{d}\right)-\left(\Delta A-\Delta B K_{x}\right) x_{m}\left(t-t_{d}\right)-\Delta B K_{r} r\left(t-t_{d}\right)
$$


The MIMO phase and time margins can be estimated by

$$
\begin{gathered}
\phi=\cos ^{-1} \frac{\bar{\mu}(A+\Delta A)+\bar{\mu}\left(j\left[(B+\Delta B) K_{x}-\frac{1}{v} P^{-1} A_{m}^{\top} P\right]\right)}{\left\|(B+\Delta B) K_{x}-\frac{1}{v} P^{-1} A_{m}^{\top} P\right\|} \\
t_{d m}=\frac{\phi}{\bar{\mu}(-j A-j \Delta A)+\left\|(B+\Delta B) K_{x}-\frac{1}{v} P^{-1} A_{m}^{\top} P\right\|}
\end{gathered}
$$

where $\bar{\mu}$ as the maximum eigenvalue of a symmetric part of a general complex matrix $C$

$$
\bar{\mu}(C)=\lambda_{\max }\left(\frac{C+C^{*}}{2}\right)
$$

where $C^{*}$ is the complex conjugate of $C$.

Utilizing this equation, the free design parameter $v$ can then be estimated to provide a measure of a time delay margin in an asymptotic sense. Similarly, the free design parameter $v$ can also be estimated to guarantee stability robustness of the predictor model adaptive laws.

Example: Consider a stable first-order plant coupled with a second-order unmodeled plant with two highly damped poles which could represent a structural mode

$$
\begin{gathered}
\dot{x}=-x+2 u-0.1 y+0.1 w(t) \\
\ddot{y}+2 \zeta \omega_{n} \dot{y}+\omega_{n} y=7 x
\end{gathered}
$$

where $\zeta=5, \omega_{n}=10$, and $-1 \leq w(t) \leq 1$ is a white noise signal representing a sensor noise source.

The reference model is

$$
\dot{x}_{m}=-2 x_{m}+2 r
$$

where the reference command is a step input with a small sinusoidal variation at the same frequency as $\omega_{n}$.

$$
r=1+0.5 \sin \omega_{n} t
$$

The controller is given by

$$
u=-\Theta^{\top} \Phi
$$

where $\Phi(t)=\left[\begin{array}{ll}x(t) & r(t)\end{array}\right]^{\top}$ and $\Theta(t)=\left[\begin{array}{ll}\theta_{x}(t) & \theta_{r}(t)\end{array}\right]^{\top}$ is updated by

$$
\dot{\Theta}=-\Gamma\left(\Phi e+v \Phi \Phi^{\top} \Theta\right)
$$

with $e(t)=x_{m}(t)-x(t), \Theta(0)=0$, and $\Gamma=100 I$.

The conventional MRAC, i.e., $v=0$, causes $\Theta(t)$ and $x(t)$ to be on the verge of instability as shown in Figs. 1 and 2 even though the open-loop plant is stable.

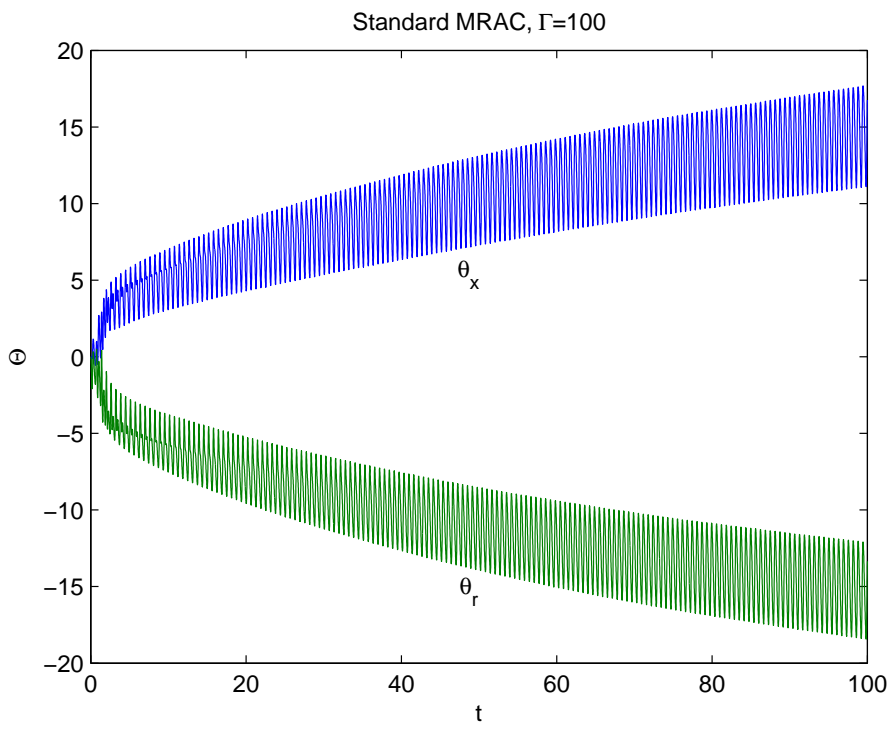


Fig. 1 - $\Theta(t)$ with MRAC, $v=0$

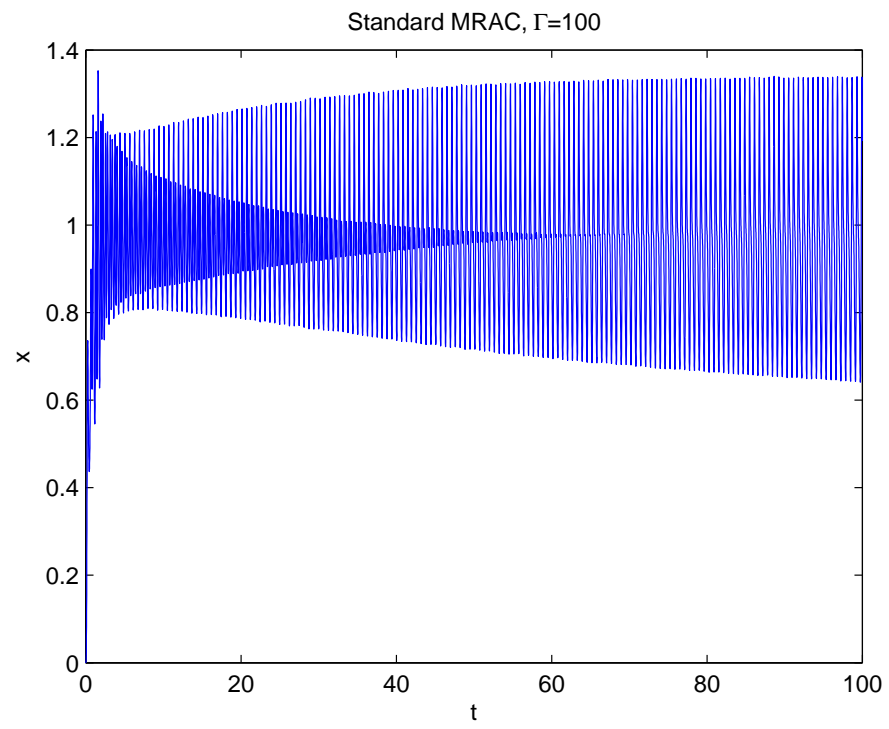

Fig. 2 - Output with MRAC, $v=0$

A value of $v=0.8$ is chosen, the optimal control modification is able to produce a stabilizing controller, as seen in Fig. 3. Thus, this illustrates the robustness of the optimal control modification. As a comparison, the $\varepsilon$-modification is used instead for the update law

$$
\dot{\Theta}=-\Gamma(\Phi e+\mu|e| \Theta)
$$

with $\mu=0.8$. The adaptive signal is stable as shown in Fig. 4 .

The asymptotic tracking error equation for fast adaptation is

$$
u^{(0)} \rightarrow \frac{e^{(0)}}{v}
$$

The asymptotic closed-loop plant can be expressed as

$$
\begin{gathered}
s x=-x+2 \frac{x_{m}-x}{v}-\frac{0.7 x}{s^{2}+2 \zeta \omega_{n} s+\omega_{n}^{2}} \\
\left(s+1+\frac{2}{v}+\frac{0.7}{s^{2}+2 \zeta \omega_{n} s+\omega_{n}^{2}}\right) x^{(0)}=\frac{4 r}{v(s+2)}
\end{gathered}
$$

As $t \rightarrow \infty$

$$
\frac{x^{(0)}}{r} \rightarrow \frac{2 \omega_{n}^{2}}{(v+2) \omega_{n}^{2}+0.7 v}
$$

Figure 3 illustrates the linear mapping between $x^{(0)}$ and $r$ for three different multipliers $0.1,0.5$, and 1.0 that scale the input signal. The predicted asymptotic ratio of $x^{(0)}$ to $r$ is 0.7129 , which agrees reasonably well with the simulation results. Fig. 4 shows response due to the $\varepsilon$-modification which produces a nonlinear mapping between $x^{(0)}$ and $r$. 


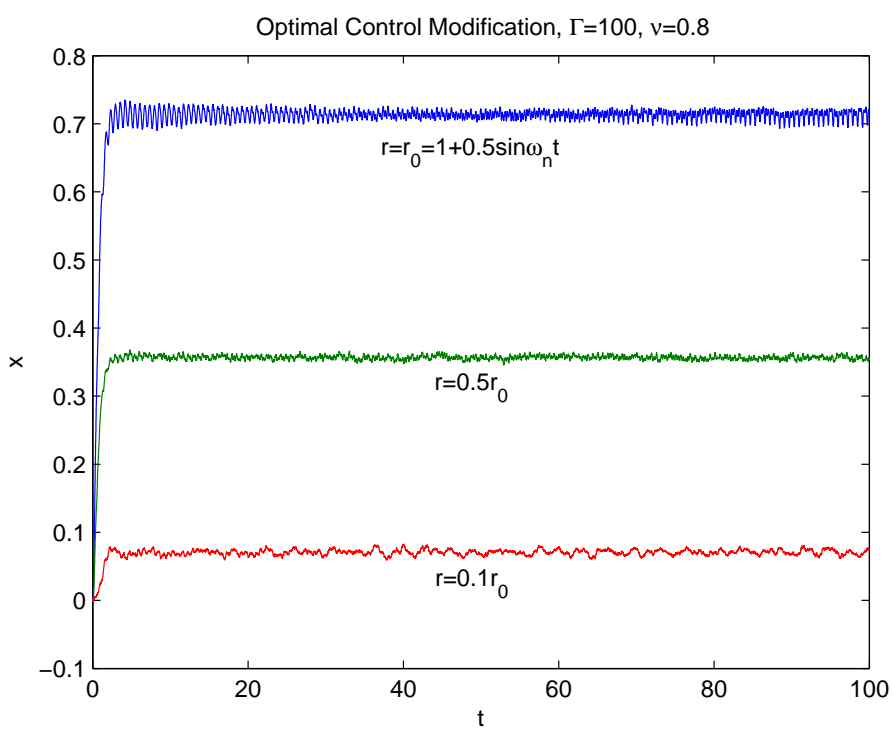

Fig. 3 - Output with Optimal Control Modification, $v=0.8$

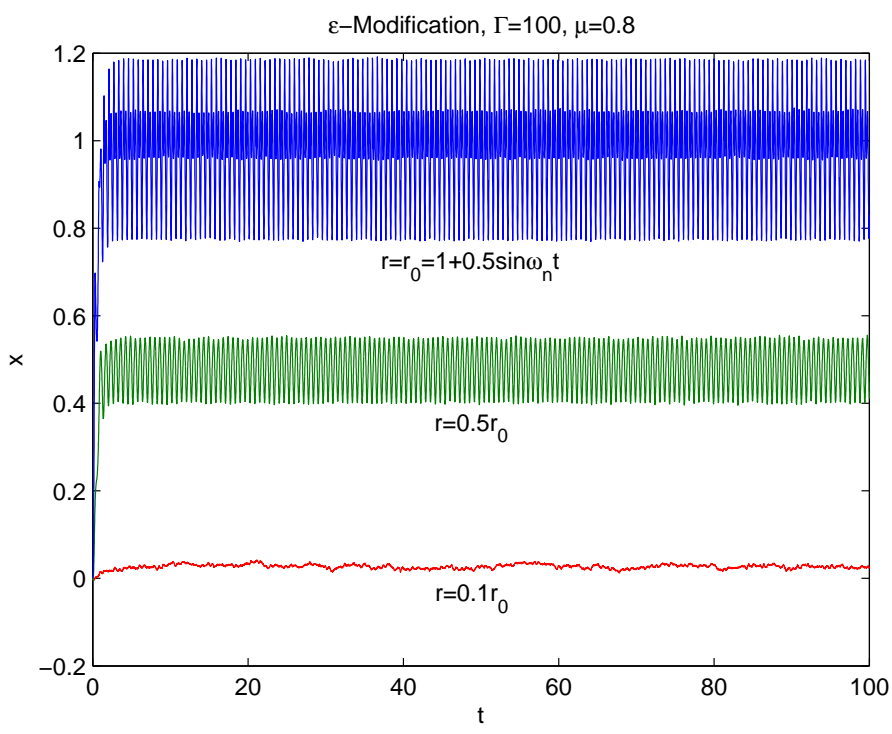

Fig. 4 - Output with $\varepsilon$ - Modification, $\mu=0.8$

\section{Flight Control Simulation}

Consider a longitudinal pitch dynamical model of an aircraft

$$
\left[\begin{array}{ccc}
m V+\frac{C_{L_{\dot{\alpha}}} \bar{q} S \bar{c}}{2 V} & 0 & 0 \\
0 & 1 & 0 \\
-\frac{C_{m_{\dot{\alpha}}} \bar{q} S \bar{c}^{2}}{2 V} & 0 & I_{y y}
\end{array}\right]\left[\begin{array}{c}
\dot{\alpha} \\
\dot{\theta} \\
\dot{q}
\end{array}\right]=\left[\begin{array}{ccc}
m g \gamma-C_{L_{\alpha}} \bar{q} S & -m g \gamma & m V-\frac{C_{L_{q}} \bar{q} S \bar{c}}{2 V} \\
0 & 0 & 1 \\
C_{m_{\alpha}} & 0 & \frac{C_{m_{q}} \bar{q} \bar{c}^{2}}{2 V}
\end{array}\right]\left[\begin{array}{c}
\alpha \\
\theta \\
q
\end{array}\right]+\left[\begin{array}{c}
-C_{L_{\delta_{e}}} \\
0 \\
C_{m_{\delta_{e}}}
\end{array}\right] \delta_{e}
$$

A numerical model for a full-scale generic transport model (GTM) at Mach 0.8 and 30,000 ft with the flight path 
angle $\gamma=0$ is given by

$$
\left[\begin{array}{c}
\dot{\alpha} \\
\dot{\theta} \\
\dot{q}
\end{array}\right]=\underbrace{\left[\begin{array}{ccc}
-0.7018 & 0 & 0.9761 \\
0 & 0 & 1 \\
-2.6923 & 0 & -0.7322
\end{array}\right]}_{A}\left[\begin{array}{c}
\alpha \\
\theta \\
q
\end{array}\right]+\underbrace{\left[\begin{array}{c}
-0.0573 \\
0 \\
-3.5352
\end{array}\right]}_{B} \delta_{e}
$$

A desired reference model of the pitch attitude is given by

$$
\ddot{\theta}_{m}+2 \zeta \omega_{n} \dot{\theta}_{m}+\omega_{n}^{2} \theta_{m}=\omega_{n}^{2} r
$$

where $\zeta=0.85$ and $\omega_{n}=1.5 \mathrm{rad} / \mathrm{sec}$ are chosen to give a desired handling characteristic.

Let $x=\left[\begin{array}{ccc}\alpha & \theta & q\end{array}\right]^{\top}$ and $u=\delta_{e}$. A nominal controller is designed as $u_{n o m}=-K_{x} x-k_{r} r$ where $K_{x}=$ $\frac{1}{b_{3}}\left[\begin{array}{lll}a_{31} & \omega_{n}^{2} & 2 \zeta \omega_{n}+a_{33}\end{array}\right]=\left[\begin{array}{lll}0.7616 & -0.6365 & -0.5142\end{array}\right]$ and $k_{r}=-\frac{1}{b_{3}} \omega_{n}^{2}=0.6365$. The closed-loop eigenvalues are -0.6582 and $-1.2750 \pm 0.7902 i$. The nominal closed-loop plant is then chosen to be the reference model as

$$
\underbrace{\left[\begin{array}{c}
\dot{\alpha} \\
\dot{\theta} \\
\dot{q}
\end{array}\right]_{m}}_{\dot{x}_{m}}=\underbrace{\left[\begin{array}{ccc}
-0.6582 & -0.0365 & 0.9466 \\
0 & 0 & 1 \\
0 & -2.2500 & -2.5500
\end{array}\right]}_{A_{m}} \underbrace{\left[\begin{array}{c}
\alpha \\
\theta \\
q
\end{array}\right]}_{x_{m}}+\underbrace{\left[\begin{array}{c}
0.0162 \\
0 \\
1
\end{array}\right]}_{B_{m}} r
$$

Suppose the control effectiveness is reduced by $50 \%$, i.e., $\Lambda=0.5$ and the matched uncertainty is represented by $\Omega^{* \top}=\left[\begin{array}{lll}0 & 0 & -0.2071\end{array}\right]$ which represents $11 \%$ increase in pitch damping and $q$ contribution to $\dot{\alpha}$.

Figures 5 is a plot of estimates of phase and time delay margins from the asymptotic solution computed from Eqs. (82) and (83) as a function of $v$. Note that the phase margin with $\Delta A$ and $\Delta B$ decreases steadily as $v$ increases, while the time delay margin reaches a maximum at about $v=1$. Thus, for practical design purposes, $v$ should be kept between 0 and 1. A large value of $v$ produces a better time delay margin, but also results in a poorer steady-state tracking.

Suppose a requirement for a $0.02 \mathrm{sec}$ time delay margin is imposed on the flight control design. Then from Fig. 5, a value of $v=0.2$ is selected to provide a sufficient time delay margin of about 0.049 sec or about 60 deg phase margin. It should be noted that the time delay margin as computed from Eq. (83) is a conservative estimate corresponding to $\Gamma_{\Theta} \rightarrow \infty$. So the actual time delay margin for a finite value of $\Gamma_{\Theta}$ should be greater than the estimated value.
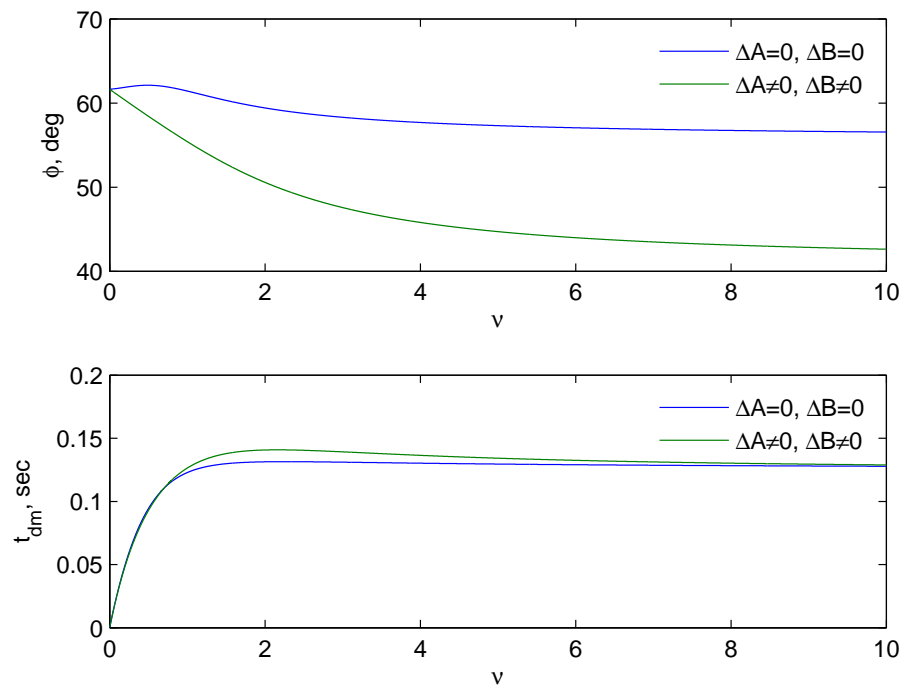

Fig. 5 - Phase and Time Delay Margin Estimates 
Figures 6,7 , and 8 show the $\alpha, \theta$, and $q$ responses due to the baseline controller. With no adaptation, the plant is not able to track the reference model very well. For the standard MRAC, an adaptive gain of $\Gamma_{\Theta}=10000 I$ is used to enable a tight tracking of the pitch attitude reference signal. For parameter estimation, adaptive gains of $\Gamma_{\Omega}=\Gamma_{\Lambda}=1000 I$ are used. Both the $\alpha$ and $\theta$ responses track the reference signals quite well. However, the $q$ response is highly oscillatory during the first $15 \mathrm{sec}$. The numerical evidence of the time delay margin of the system with the standard MRAC is observed to be $0.04 \mathrm{sec}$. Figure 8 shows the responses with the optimal control modification for the same adaptive gains. The $q$ response is significantly improved with almost no noticeable high frequency oscillations. The observed numerical evidence of the time delay margin with the optimal control modification is $0.09 \mathrm{sec}$, which is a significant improvement over that for the standard MRAC.
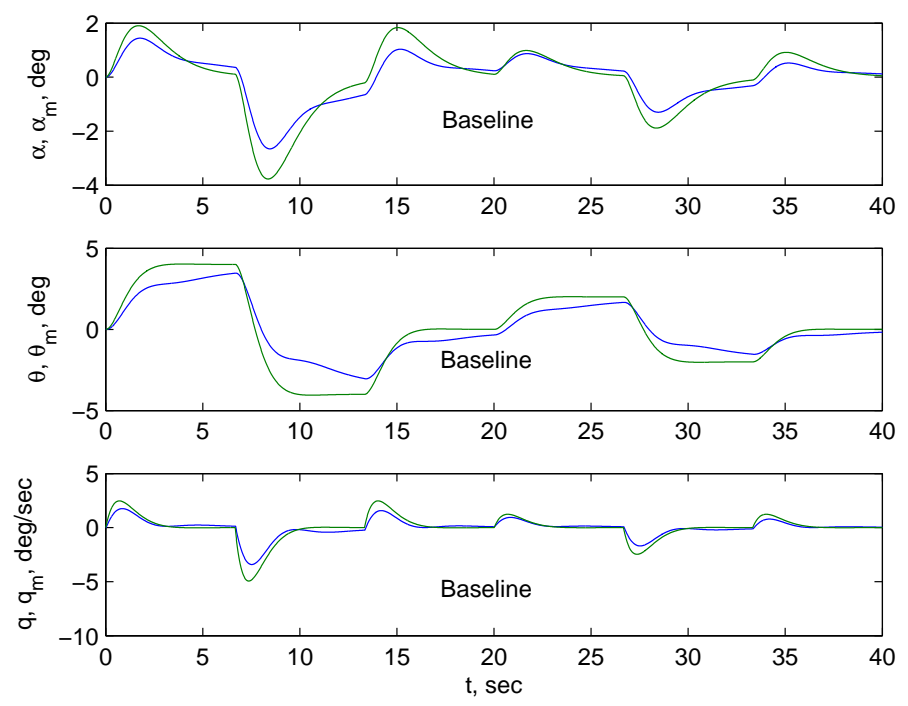

Fig. 6 - $\alpha, \theta$, and $q$ Responses with Baseline Controller
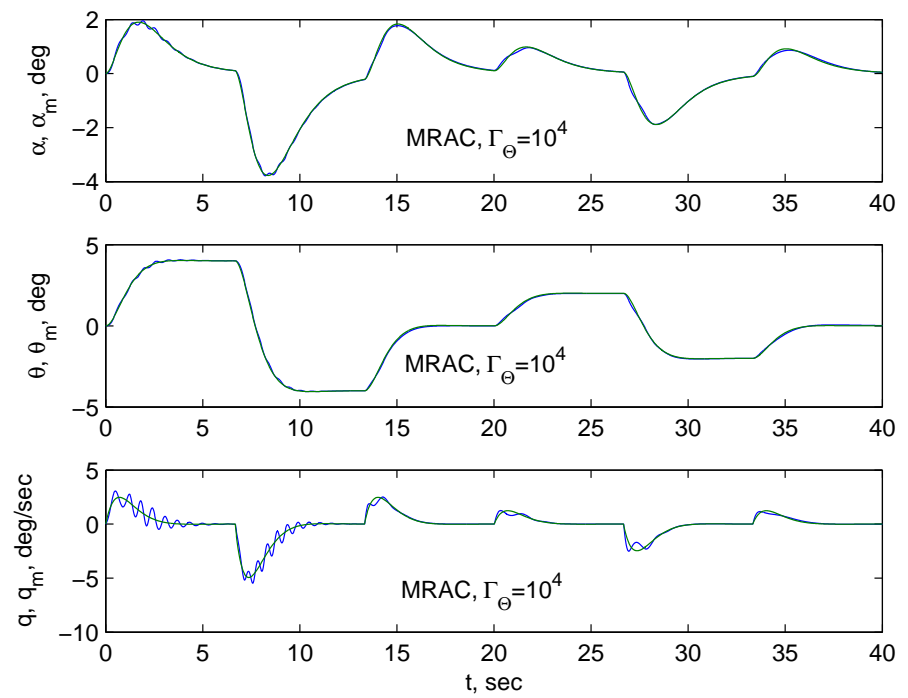

Fig. 7 - $\alpha, \theta$, and $q$ Responses with Standard MRAC 

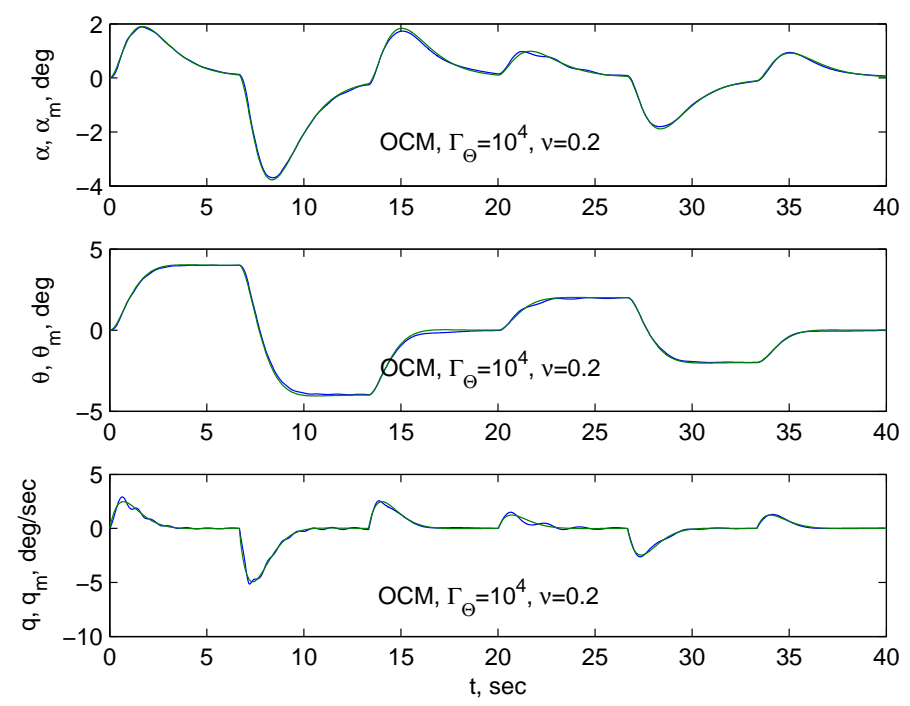

Fig. $8-\alpha, \theta$, and $q$ Responses with Optimal Control Modification (OCM)
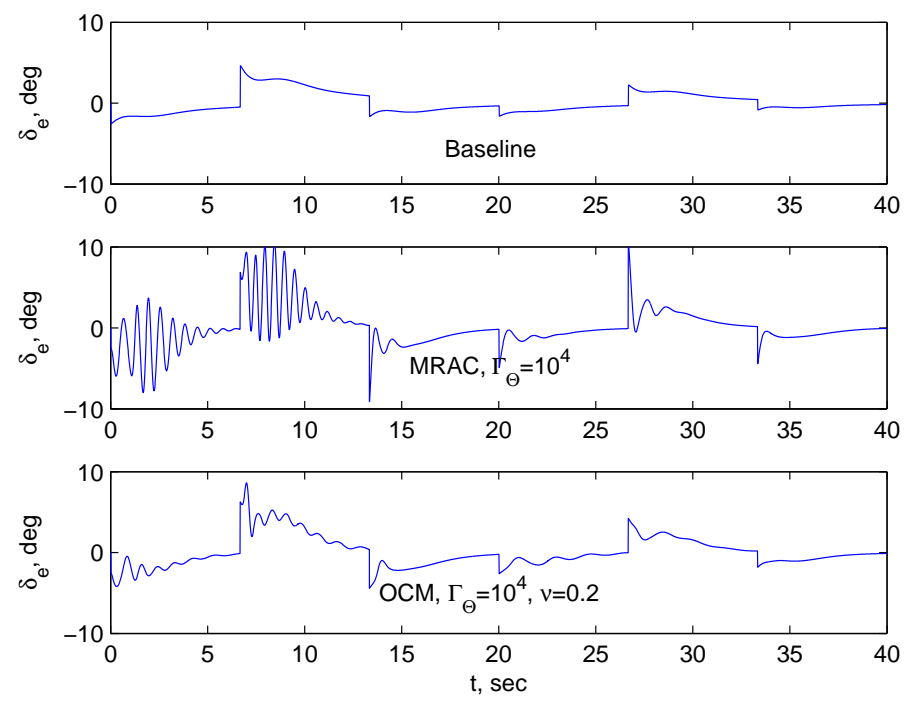

Fig. 9 - Elevator Deflection

Figure 9 shows the control signals produced by the baseline controller, standard MRAC, and optimal control modification. The control signal produced by the standard MRAC exhibits large amplitude, high frequency oscillations in the first $15 \mathrm{sec}$. In contrast, this behavior is not seen with the optimal control modification which produces a wellbehaved control signal.

\section{Conclusions}

This study presents a verifiable model-reference adaptive control method based on an optimal control formulation for linear uncertain systems. A predictor model is formulated to enable a parameter estimation of the system parametric uncertainty. The adaptation is based on both the tracking error and predictor error. Using a singular perturbation argument, it can be shown that the closed-loop system tends to a linear time invariant model asymptotically under an assumption of fast adaptation. A stability margin analysis is given to estimate a lower bound of the time delay margin using a matrix measure method. Using this analytical method, the free design parameter $v$ of the optimal control modification adaptive law can be determined to meet a specification of stability margin for verification purposes. 
A simple scalar system is used to demonstrate the asymptotic linearity of the optimal control modification adaptive law when the system exhibits a linear input-output mapping under fast adaptation. A flight control application for a generic transport aircraft is studied. The simulation results demonstrate the effectiveness of the optimal control modification, which shows its ability to suppress high frequency oscillations in the control signal, thereby enabling improved robustness. The present method may provide a path toward addressing technical challenges with verification and validation of adaptive control. The ability to verify an adaptive control algorithm is an important step toward future certification of adaptive control for safety-critical systems.

\section{References}

\footnotetext{
${ }^{1}$ Nguyen, N., and Jacklin, S., "Neural Net Adaptive Flight Control Stability, Verification and Validation Challenges, and Future Research," Workshop on "Applications of Neural Networks in High Assurance Systems", International Joint Conference on Neural Networks, August 2007.

${ }^{2}$ Jacklin, S. A., Schumann, J. M., Gupta, P. P., Richard, R., Guenther, K., and Soares, F., "Development of Advanced Verification and Validation Procedures and Tools for the Certification of Learning Systems in Aerospace Applications," AIAA Infotech@ Aerospace Conference, AIAA-2005-6912, October 2005.

${ }^{3}$ Sang, Q. and Tao, G., "Gain Margins of Multivariable MRAC Systems", 47th IEEE Conference on Decision and Control, December 2008.

${ }^{4}$ Nguyen, N. T. and Boskovic, J. D., "Bounded Linear Stability Margin Analysis of Nonlinear Hybrid Adaptive Control", American Control Conference, June 2008.

${ }^{5}$ Cao, C. and Hovakimyan, N., "Guaranteed Transient Performance with $\mathscr{L}_{1}$ Adaptive Controller for Systems with Unknown Time-varying Parameters and Bounded Disturbances: Part I," American Control Conference, June 2007.

${ }^{6}$ Cao, C. and Hovakimyan, N., "Stability Margins of $\mathscr{L}_{1}$ Adaptive Controller: Part II," American Control Conference, June 2007.

${ }^{7}$ Nguyen, N., Krishnakumar, K., and Boskovic, J., "An Optimal Control Modification to Model-Reference Adaptive Control for Fast Adaptation," AIAA Guidance, Navigation, and Control Conference, AIAA 2008-7283, August 2008.

${ }^{8}$ Calise, A., Yucelen, T., Muse, J., Yang, B., ”A Loop Recovery Method for Adaptive Control," AIAA Guidance, Navigation, and Control Conference, AIAA-2009-5967, August 2009.

${ }^{9}$ T. Yucelen and A. Calise, "A Kalman Filter Optimization Approach to Direct Adaptive Control," AIAA Guidance, Navigation, and Control Conference, AIAA-2009-5858, August 2009.

${ }^{10}$ Volyanskyy, K., Calise, A., and Yang, B., "A Novel Q-Modification Term for Adaptive Control," American Control Conference, June 2006.

${ }^{11}$ Lavretsky, E., "Combined/Composite Model Reference Adaptive Control," IEEE Transactions on Automatic Control, Vol. 54, No. 11, November 2009.

${ }^{12}$ Muse, J. and Calise, A., “ " $\mathscr{H}_{\infty}$ Neural Network Adaptive Control," American Control Conference, June 2010.

${ }^{13}$ Ioannu, P.A. and Sun, J., Robust Adaptive Control, Prentice-Hall, 1996.

${ }^{14}$ Bryson, A.E. and Ho, Y.C., Applied Optimal Control: Optimization, Estimation, and Control, John Wiley \& Sons Inc., 1979.

${ }^{15}$ Khalil, H. K., Nonlinear Systems, Prentice-Hall, 2002.

${ }^{16}$ Kokotovic, P., Khalil, H., and O'reilly, J., Singular Perturbation Methods in Control: Analysis and Design, Society for Industrial and Applied Mathematics, 1987.

${ }^{17}$ Ardema, M., "Computational Singular Perturbation Method for Dynamical Systems”, AIAA Journal of Guidance, Control, and Dynamics, Vol. 14, 661-663, 1981.
} 